\title{
Colonic stricture in a boy with cystic fibrosis
}

\author{
PS Ong, PJ Oades, A Bush, RJ Brereton
}

\begin{abstract}
Summary
Many problems may arise within the gastrointestinal tract of patients with cystic fibrosis. We report a new cause of subacute intestinal obstruction due to a fibrotic stricture of the ascending colon in a child with cystic fibrosis. Treatment was with a right hemicolectomy. There has been no recurrence after nine months follow-up. Recent similar cases suggest that this new pathology is linked to the use of enteric-coated high-strength pancreatin microspheres.
\end{abstract}

Keywords: cystic fibrosis, colonic fibrotic stricture, pancreatic enzymes

Abdominal pain and discomfort are frequent diagnostic problems in patients with cystic fibrosis and a variety of diseases may be responsible. Distal intestinal obstruction syndrome occurs in approximately $15 \%$. This causes pain in association with a mass in the right lower quadrant due to inspissated material in the ileocaecal region. It may be mimicked by or coexist with other diseases (see box). ${ }^{1} \mathrm{We}$ report a boy with cystic fibrosis but without any history of abdominal surgery who developed intestinal obstruction with a right lower quadrant mass from a newly recognised pathology. A series of similar cases have recently been reported, all were boys taking the new highstrength pancreatin preparations. ${ }^{2}$

\section{Case report}

Cystic fibrosis was diagnosed in a four-month old boy following a bronchiolitic illness (duplicate sweat sodium concentrations of more than $70 \mathrm{mmol} / \mathrm{l}$ on more than $100 \mathrm{mg}$ of sweat; homozygous for Delta F508). There had been no neonatal problems. He had pancreatic insufficiency but thrived on pancreatic enzyme supplements. When two years old he started on a high-strength pancreatin supplement, $\mathrm{Nu}$ Royal Brompton

National Health \&

Lung Hospital,

London, UK

PJ Oades

A Bush

\section{Correspondence to}

Dr PS Ong, Department of Histopathology, Charing

Cross Hospital, Fulham

Palace Road, London

W6 6RF, UK

Accepted 16 November 1994
- inspissated materia

- volvulus

- Crohn's disease

- stricture from pancreatin supplement usage trizyme 22 (22 $000 \mathrm{U}$ lipase, $19800 \mathrm{U}$ amylase and $1100 \mathrm{U}$ protease per capsule), initially consuming 15 capsules per day.

At age of 2 years and 9 months, he developed an insidious fall in appetite associated with intermittent colicky abdominal pain. After several weeks this was followed by nocturnal vomiting, less frequent bowel actions, which were of normal consistency and his weight fell from the $50^{\text {th }}$ to the $25^{\text {th }}$ centile. These symptoms failed to respond to either laxatives or an increased enzyme intake ( 25 capsules per day). Other medications included budesonide via Nebuhaler $(800 \mu \mathrm{g} /$ day $)$ for wheezing and several courses of co-amoxiclav (amoxycillin + clavulanic acid) for respiratory tract infections.

On examination, he was lethargic and mildly dehydrated $(<5 \%)$. His abdomen was distended with an ill-defined tender mass in the right iliac fossa. Bowel sounds were normal and no faeces or abnormalities were found on rectal examination. There was no clinical evidence of active pulmonary disease.

Investigations revealed haemoglobin 13.7 $\mathrm{g} / \mathrm{dl}$, erythrocyte sedimentation rate $4 \mathrm{~mm} / \mathrm{h}$ and leucocyte count $13.4 \times 10^{9} / 1$, which were all within normal range for his age. An abdominal radiograph showed dilated loops of small bowel (figure 1) but no fluid levels and no features to suggest distal intestinal obstruction syndrome. An abdominal ultrasound identified thickened bowel wall $(7 \mathrm{~mm})$ in the region of the caecum and a barium enema demonstrated a featureless transverse colon leading to a narrow and irregular ascending colon which was slow to fill with contrast (figure 2).

Conservative management failed so he underwent laparotomy. A thickened ileocaecal valve, caecum and proximal $8 \mathrm{~cm}$ of ascending colon were resected and a primary ileocolic anastomosis was performed. The bowel was not discoloured but the lumen was narrowed by concentric thickening of the wall. The mucosa had a mild cobblestone appearance without ulceration (figure 3 ) and the mesentery was normal.

Histology showed characteristic changes of cystic fibrosis in the mucosa, especially in the small bowel (figure 4). The thickening and cobblestone appearance were caused by extensive submucosal fibrosis which reached the line of resection. Fibrosis was less marked in the mucosa and serosa. The muscularis propria was relatively intact. There was evidence of low-grade inflammation confined to the mucosa (figure 5). Granulomata were not seen 


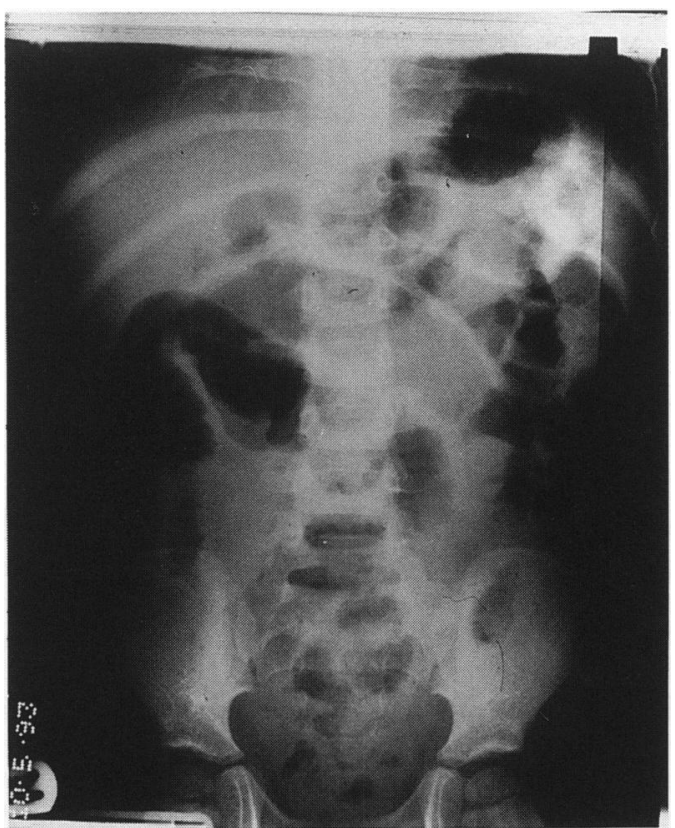

Figure 1 Plain abdominal radiograph showing dilated loops of bowel, but no faecal loading. Gas in the right lower quadrant has an 'apple core' appearance

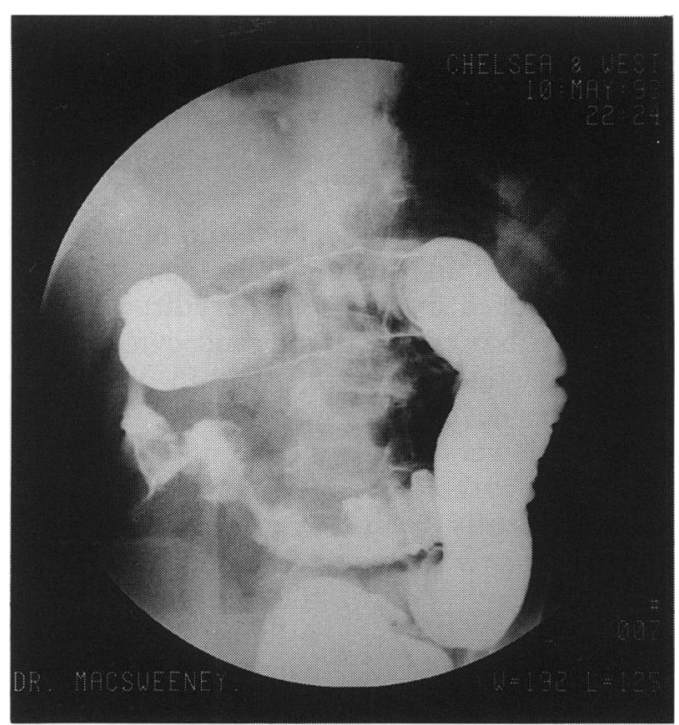

Figure 2 Water-soluble contrast barium enema showing a normal rectum, sigmoid and descending colon and an irregular stricture of the ascending colon with coarse mucosal folds. There is no faecal loading. The transverse colon is featureless and from the hepatic flexure, contrast progressed slowly under pressure to reach the small bowel

and stains for amyloid and haemosiderin were negative. Blood vessels and lymph nodes were normal. The appendix contained abundant mucus but was otherwise normal.

His postoperative course was uncomplicated. His appetite improved and his bowel frequency returned to normal. Nine months later he remained well with no abdominal symptoms and is thriving.

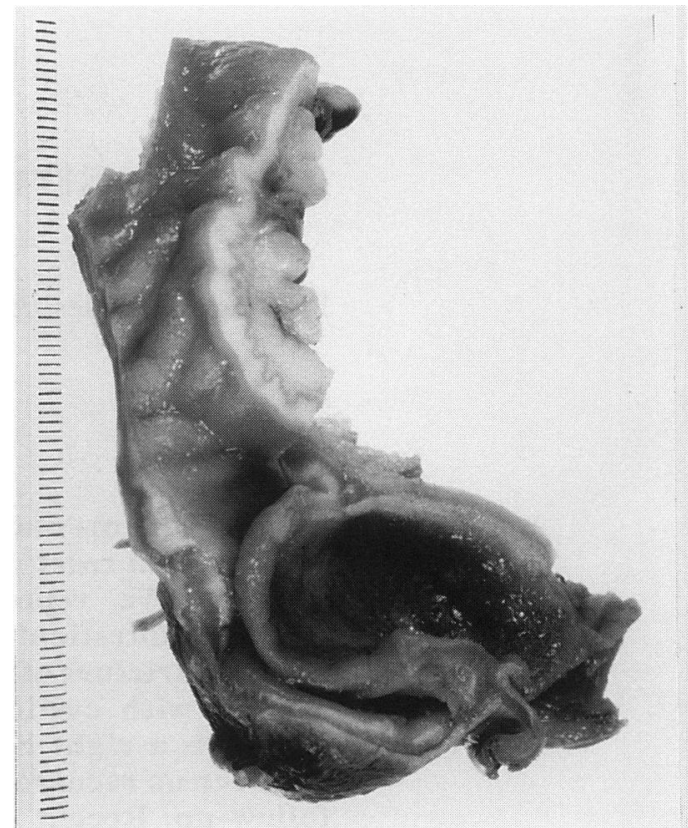

Figure 3 Macroscopic view of the terminal ileum, caecum and ascending colon. The mucosa has a cobblestone appearance but without ulceration. The pale band of submucosal fibrosis is prominent. Scale shown is in millimeter increments

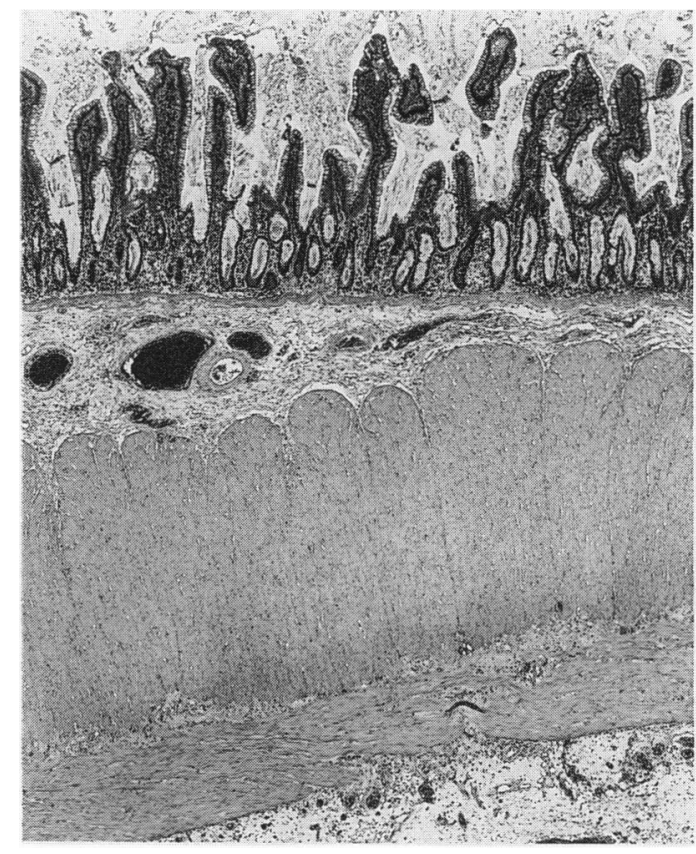

Figure 4 Small bowel histology showing characteristic features of cystic fibrosis, namely abundant mucus between villi and within slightly distended crypts. Haematoxylin and eosin $\times 30$

\section{Discussion}

This child presented with subacute intestinal obstruction and a mass in the right iliac fossa due to a long fibrotic stricture of the ascending colon. At presentation a primary diagnosis of distal intestinal obstruction syndrome was excluded by radiology. The differential diagnosis then included recurrent intussusception or 


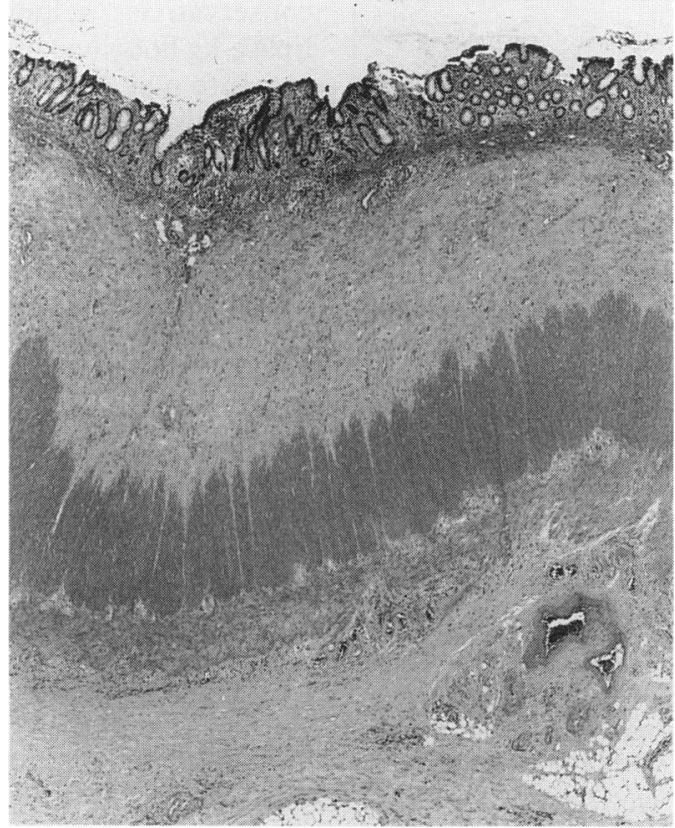

Figure 5 Histology of colonic stricture. There is mild inflammation in the mucosa and marked submucosal fibrosis. The muscularis propria is relatively normal. Haematoxylin and eosin $\times 30$

Crohn's disease, both of which are more common in children with cystic fibrosis than in the general population. Other considered possibilities included a retrocolic appendix mass partly suppressed by the co-amoxiclav, ileocaecal tuberculosis, or a tumour, but these were excluded at operation and by histology.

Generalised intestinal fibrosis, leading to mechanical ileus and malabsorption, has been reported in several situations (see box). ${ }^{3-5}$ In horses, environmental factors have been implicated in a similar process. ${ }^{6}$ Localised colonic fibrotic strictures may occur after ischaemic colitis, usually at a vascular watershed near the splenic flexure. The history in this case is not of an acute ischaemic event and the blood vessels were histologically normal. It is conceivable that recurrent intussusception could cause venous congestion and localised ischaemic damage but no intussusception was found on radiographic imaging or at operation and the relative absence of fibrosis in the muscularis propria or evidence of old haemorrhage on histological examination make this diagnosis unlikely. Crohn's disease may result in localised strictures following intense ulceration and inflammation but it is rare under the age of

\begin{tabular}{|l|}
\hline Intestinal fibrotic strictures: causes \\
\hline - scleroderma \\
- high-dose irradiation \\
- non-steroidal anti-inflammatory drugs \\
- folloric-coated potassium supplements \\
- Crohn's dischaemic colitis \\
\hline
\end{tabular}

nine years. There were no ulcers, fissures, transmural inflammation or granulomas which are classical histological features of Crohn's disease. Similarly, the short history, the normal mesentery, erythrocyte sedimentation rate and haemoglobin, all make inflammatory bowel disease unlikely.

Pseudotumoral thickening of the ascending colon with similar radiographic findings has been reported in an adult with cystic fibrosis ${ }^{7}$ but no histological features were described. However, a recent report describes five cases of colonic strictures in boys with cystic fibrosis who had taken high-strength pancreatic enzymes for $12-15$ months. ${ }^{2}$ The pathological changes were considered to be the result of either pressure-induced ischaemia from an intra-luminal faecal mass or direct toxicity. The authors favoured the former mechanism and postulated that the new preparations lower the lipid content of mucus and faecal material encouraging its dehydration, so leading to increased viscosity and accumulation in the colon.

Oral mucosal irritation with contact bleeding and peri-anal soreness has been associated with the use of pancreatic enzymes. ${ }^{8}$ Therefore, a similar chronic reaction due to inappropriate enzyme activity in the ascending colon may account for the findings in this case. Indeed, stasis of faecal material in the ileocolic region is common in cystic fibrosis and we have observed intact enzyme microspheres in stools of some young patients despite the in vitro reports of rapid dissolution of enteric-coated pancreatin microspheres. ${ }^{9}$ However, any mechanism involving, or association with, high-strength pancreatin must remain speculative. Our patient remained on high-strength enzymes for 7 months after operation when the possibility of adverse reaction was identified. $\mathrm{He}$ is now taking standard strength preparations and ultrasound scan has revealed no evidence of further stricture, although his transverse colon is thickened (3.4 mm).

There is no doubt that the introduction of pancreatic enzyme supplements has contributed to the improved nutritional status and increased survival of patients with cystic fibrosis. However, patients frequently exceed the recommended dose, tailored according to abdominal symptoms, stool consistency, and odour. The high-strength enzyme capsules have been shown to improve the coefficient of fat absorption and enable patients to reduce capsule intake significantly, but ultimately patients tend to take a higher total daily dose of enzyme units.

At present, no colonic strictures have been reported in association with standard strength pancreatin preparations. It seems appropriate for patients with cystic fibrosis to change back to the standard preparations and to adjust doses to the minimum required for adequate fat absorption unless there are good reasons for persisting with the high-strength preparation. Further investigation is required to determine the mechanism by which fibrosis may be provoked. It is not clear why there is a male predominance. In Germany, where high-dose 


\section{Learning points}

- distal intestinal obstruction occurs in $15 \%$ of patients with cystic fibrosis

- enteric-coated high-strength pancreatin microspheres have been linked with the development of fibrotic strictures of the ascending colon

enzyme preparations have been used since 1986, there are no known cases, but dosing is

1 Dalzell AM, Heaf DP, Carty H. Pathology mimicking dista intestinal obstruction syndrome in cystic fibrosis. Arch Dis Child 1990; 65: 540-1.

2 Smyth RL, Van Velzen D, Smyth AR, Lloyd DA, Heaf DP. Strictures of ascending colon in cystic fibrosis and highStrictures of ascending colon in cystic fibrosis and high
strength pancreatic enzymes. Lancet 1994; 343: 85-6.

3 Daum R, Nutzendel W, Roth H, Zacharia Z. Motility malfunction of the gastrointestinal tract by rare disease fibrosis of the intestinal wall. Prog Paediatr Surg 1989; 24: 193-201

4 Levi S, de Lacey G, Price AB, Gumpel MJ, Levi AJ Bjarnason I. 'Diaphragm-like' strictures of the small bowe in patients treated with non-steroidal anti-inflammatory drugs. Br F Radiol 1990; 63: 186-9.

5 Trechot P. Reasons for the removal of an enteric-coated slow-release potassium preparation from the marker in France. Am $\mathcal{f}$ Gastroenterol 1990; 85: 330-1. conservative, seldom exceeding $40000 \mathrm{U}$ lipase/kg body weight daily. ${ }^{10}$ One worry is that stricture may represent one end of a spectrum of fibrosis whereas less severe forms could give rise to reduced gut motility, as described in small bowel fibrosis. Colonic strictures should be added to the list of differential diagnoses in patients with cystic fibrosis presenting with abdominal pain and vomiting. Investigation by abdominal radiography, abdominal ultrasonography and possibly barium enema to look for bowel wall thickening and lumenal narrowing should be considered in such circumstances.

6 Traub-Dargatz JL, Schultheiss PC, Kiper MC, et al. Intestinal fibrosis with partial obstruction in five horses and two ponies. $¥$ Am Vet Med Assoc 1992; 201: 603-7.

7 Feigelson J, Girault F, Montagne JP, Faure C. Late and unusual intestinal features in cystic fibrosis - pseudotumoral intestinal thickening. Acta Univ Carolinae Med 1990; 36: intestinal

8 Oades PJ, Marchant J, Sheppard M, Bush A. Changing to high-dose pancreatic enzymes. Pediatr Pulmonol 1992; 286 (Suppl 8): 312 (abstract)

9 Atkinson SN. A comparative study of enzyme activity, acid resistance and dissolution characteristics of four entericcoated microsphere preparations of pancreatin. Eur $\mathcal{f}$ Clin Res 1991; 1: 37-45.

10 Taylor CJ. Colonic strictures in cystic fibrosis. Lancet 1994; 343: 615-6. 